\title{
ELABORACIÓN, CARACTERIZACIÓN ELECTROQUÍMICA Y ESTUDIO MOTRIZ DE UN ACTUADOR TRICAPA
}

\author{
Saúl Bertel Hoyosª, Jairo Segura Bermúdez ${ }^{\text {b }}$ Álvaro Arrietac ${ }^{\mathrm{c}}$, Yamid Núñez ${ }^{\mathrm{a}}$
}

\begin{abstract}
RESUMEN
En este trabajo se presenta la obtención de una biopelícula con propiedades semiconductoras, desarrollada a partir de almidón de yuca dopada con perclorato de litio y plastificantes como glicerina y glutaraldehido, que le proporcionaron propiedades eléctricas y mecánicas necesarias para generar movimiento. Se empleó la síntesis electroquímica de Polipirrol $(P P y)$ con el fin de obtener las capas externas para la constitución de un actuador tricapa (PPy/biopelícula/PPy) capaz de responder a estímulos eléctricos y funcionar en el aire. El dispositivo fue sometido a potenciales recurrentes que generaron la óxido/reducción del $P P y$ permitiendo el movimiento del actuador. Posteriormente, con el objeto de describir algunas de sus propiedades fue caracterizado mediante espectroscopía de impedancia electroquímica (EIS), voltametría cíclica, técnicas muy usuales en la actualidad para el estudio de este tipo de materiales. Por otro lado, se realizó un estudio motriz del músculo artificial tricapa, teniendo en cuenta las respuestas a pulsos eléctricos.
\end{abstract}

Palabras clave: Biopelícula, Polipirrol, actuador tricapa, músculo artificial.

\section{ELABORATION, ELECTROCHEMICAL CHARACTERIZATION AND MOTION STUDY OF A THREE-LAYER ACTUATOR}

\begin{abstract}
In this work is presented the development of a biofilm with semiconducting properties, derived from cassava starch and plasticizers such as glycerin and glutaraldehyde and doped with lithium perchlorate, which provided the electrical and mechanical properties needed to achieve the desired motion. Electrochemically synthesized polypyrrole (PPy) was used in order to obtain the outer layers to constitute a three-layer actuator (PPy / biofilm / PPy) able to react to electrical stimulus and operate in air. Differents recurrent potentials were administered to the device generating the oxidation and reduction processes in the PPy, easing the movement of the actuator as a whole. In order to describe some of its properties, the actuator was characterized by electrochemical impedance spectroscopy and cyclic

\footnotetext{
a. Facultad de Ciencias Básicas, Fundación Universitaria los Libertadores, Carrera 16 \# 63a-68, Bogotá, 111211 , Colombia, sdbertelh@libertadores.edu.co.

b CEN CliSAP, Universität Hamburg, Hamburg 20095, Alemania
}

c Departamento de Biología y Química, Universidad de Sucre, Sincelejo, Colombia
\end{abstract}


voltammetry. Furthermore, a study of the trilayer muscle deflection angle was performed, considering the responses to electrical pulses.

Key words: Biofilm, Polypyrrole, actuator threelayer, artificial muscle.

\section{INTRODUCCIÓN}

En los últimos años, se ha venido investigando intensamente en la identificación y desarrollo de materiales y dispositivos capaces de imitar el funcionamiento y desempeño de los músculos naturales. Teniendo en cuenta su naturaleza y funcionamiento, se requieren materiales capaces de conducir iones y electrones. El rango del pulso eléctrico tiene que ser del orden de los $\mathrm{mV}$, que es el intervalo de potenciales que usan los músculos naturales $(\approx 175 \mathrm{mV})$. Deben experimentar una variación de volumen asociada a cambios conformacionales en su estructura. Además deben tener una estructura molecular similar, por lo que estos materiales deben tener componentes macromoleculares. De esta manera, a la hora de buscar sistemas artificiales capaces de imitar el funcionamiento de los músculos naturales, los más apropiados han sido los sistemas electroquímicos basados en polímeros conductores ${ }^{1}$.

En la última década, se han desarrollado muchas alternativas para músculos artificiales, como los geles poliméricos, pero sin duda los polímeros conductores son los que han surgido como la mejor opción. Los primeros actuadores que recibieron el nombre de músculo artificial se basan en polímeros conductores ${ }^{2}$, también denominados dispositivos electroquimiomecánicos por involucrar una reacción electroquímica en la conversión a energía mecánica.

El principio básico de este dispositivo es la oxidación/reducción electroquímica de los polímeros conductores, que lleva asociado un cambio de volumen del material. El cambio de volumen (propiedad mecánica) está asociado al grado de oxidación y éste a la carga consumida en el proceso electroquímico: propiedad electroquímica. En el proceso de oxidación, partiendo de un polímero en estado neutro, las elevadas interacciones polímero-polímero hacen que la estructura sea muy compacta. Al oxidarse y extraerse electrones de la cadena, las cargas positivas generadas en cadenas vecinas provocan fuertes repulsiones electrostáticas. Mediante variaciones conformacionales las cadenas se mueven, la estructura se abre y los contraiones de la disolución penetran en el polímero para mantener la electroneutralidad. Con los iones también penetran moléculas del disolvente y por tanto el polímero se expande, otorgándole al músculo en su movimiento la capacidad de ser detenido en cualquier momento o ser invertido desde cualquier momento.

Con el paso de los años, se han propuesto diferentes tipos de músculos artificiales. La primera generación se inició en 1955 por Katchalski y se basaba en geles poliméricos no conductores electrónicos. La dificultad que se presentaba en ese entonces era que los geles reaccionaban a muy elevados potenciales $(>20 \mathrm{~V})$, necesitaban dos electrodos metálicos auxiliares para crear el campo eléctrico requerido para su movimiento, trabajaban mediante fenómenos electrocinéticos (electroforesis y electro-osmosis), y eran denominados dispositivos electrocineto-mecánicos y su velocidad de movimiento fue relativamente baja. 
La segunda generación de músculos artificiales la constituyen los basados en polímeros conductores electrónicos intrínsecos, que fueron patentados en 1992. Con esta generación, se ha acercado a los músculos naturales en varios aspectos fundamentales: trabajan a bajo potencial $(100 \mathrm{mV}-2 \mathrm{~V})$, los músculos naturales a $60-150 \mathrm{mV}$, que es el potencial del pulso nervioso, el mismo material es conductor electrónico, iónico y es actuador y sensor de las condiciones de trabajo ${ }^{1}$.

Para transformar los movimientos conformacionales moleculares electroasistidos, en movimientos macroscópicos, en el Laboratorio de Electroquímica de la Facultad de Químicas de San Sebastián, se ideó y patentó un dispositivo de doble capa, similar a los termómetros de bilámina metálica. Al electrodo metálico, recubierto de una película de PPy electrogenerada de $15 \mu \mathrm{m}$, se le adhirió una película polimérica comercial no conductora, de $30 \mu \mathrm{m}$. La bilámina (PPy/película adherente) se desprendió del electrodo y se empleó como nuevo electrodo en una disolución acuosa.

Tradicionalmente, los músculos artificiales de la segunda generación que se reportan, funcionan en una solución acuosa electrolítica, la cual permite el paso de iones hacia y desde el polímero conductor. En pocas ocasiones se ha reportado músculos artificiales basados en polímeros conductores capaces de moverse en aire, sin embargo, los reportados sustituyen a la solución electrolítica por un polímero conductor sintético, o por un gel polimérico.

Recientemente, un equipo de investigadores de la Universidad Nacional de Taiwán fabricó un músculo artificial a partir de células de cebolla, se notó que al fluir la corriente las células de cebolla se doblan con un voltaje $0 \mathrm{~V}-50 \mathrm{~V}$ y se estiran con un voltaje $50 \mathrm{~V}-1000 \mathrm{~V}$, como un músculo "natural". Este músculo que funciona en aire solo se desplazó una distancia de 1 $\mathrm{mm}$ y como allí se menciona los voltajes son elevados comparados con los voltajes referidos a los de los músculos naturales, sin embargo representa un gran aporte al estudio y evolución de los músculos artificiales ${ }^{3}$.

No obstante, en este trabajo se presenta un dispositivo con características de músculo artificial, respondiendo a pulsos eléctricos del orden de unos cuantos voltios (0,5 V- $4 \mathrm{~V})$, es el primer músculo artificial en el mundo que emplea un polímero de almidón de yuca capaz de responder a estímulos eléctricos en el aire, barriendo distancias angulares de hasta $83,5^{\circ}$.

\section{PARTE EXPERIMENTAL}

En el desarrollo experimental se llevaron a cabo varias etapas, inicialmente, la obtención de la biopelícula, luego la síntesis del PPy y por último la obtención del dispositivo triacapa.

\section{Obtención de la biopelícula conductora}

Para la obtención de la biopelícula conductora, primero se procesó la yuca o cassava (Manihot esculenta) haciendo uso de las operaciones básicas de lavado, pelado, rallado, filtrado, decantado, secado y molienda para la obtención de almidón. 
Para elaborar las películas de almidón primero se vertió $100 \mathrm{ml}$ de agua milli-Q con pH 9,0, ya que se ha comprobado que con $\mathrm{pH}$ básicos las moléculas de amilopectina se despliegan facilitando la entrada a plastificantes en la estructura ${ }^{4}$. Luego se agregó el almidón a temperatura ambiente, se dejó en agitación constante durante 15 min hasta tener una mezcla homogénea. Una vez obtenida dicha mezcla, se vertieron los siguientes reactivos: glicerina (GLY), glutaraldehido (GLU) y perclorato de litio (LP) que proporcionaron las propiedades mecánicas de flexibilidad y conductoras respectivamente, e inmediatamente se aumentó la temperatura hasta $70{ }^{\circ} \mathrm{C}$ durante 15 minutos, posteriormente se depositó en un recipiente de teflón y se llevó a una incubadora por un periodo de 48 horas a una temperatura de $70{ }^{\circ} \mathrm{C}$. Pasado este periodo, se retiró de la incubadora y se dejó reposar a temperatura ambiente por 24 horas. Seguidamente la Síntesis del $P P y$.

\section{Síntesis de PPy}

Las películas de PPy se obtuvieron mediante la técnica de síntesis electroquímica. Para ello, se preparó una mezcla de LP y pirrol en acetonitrílo, la que finalmente se colocó en un beaker de $50 \mathrm{ml}$. Luego, empleando un sistema constituido por tres electrodos, un electrodo de trabajo (EW) en el que se depositó el polímero (polipirrol), un electrodo de referencia (ER) de Calomel saturado, y un electrodo auxiliar (EA) o contra electrodo, ubicado a los lados del EW. Como EW y EA, se usaron láminas de acero inoxidable de 2 × 2,5 cm. Posteriormente, se aplicó un potencial de $0,8 \mathrm{~V}$ durante un tiempo de $2500 \mathrm{~s}$ mediante un potenciostato/ galvanostato Parstat 2263 para generar el crecimiento de las capas de $P P y$ en el EW.

\section{Obtención del tricapa}

Se realizó un corte de 4,5 $\mathrm{cm}^{2}(3 \mathrm{~cm}$ x 1,5 cm) de biopelícula conductora, y se colocó sobre una de las caras del PPy que se encontraba en el EW. Luego de igual forma se procedió para retirar la segunda capa de $P P y$, obteniéndose así el constituyente tricapa (PPy/Biopolímero conductor/PPy).

\section{RESULTADOS Y DISCUSIÓN}

\section{Espectroscopía de impedancia electroquímica y voltametría cíclica}

La resistividad iónica del dispositivo desarrollado se determinó a partir de su espectro de impedancia, para ello, se tomó $1 \mathrm{~cm}^{2}$ del tricapa PPy/Biopolimero/PPy, que fue conectado previamente en medio de dos placas de acero inoxidable y posteriormente al potenciostato/ galvanostato Parstat 2263, Princeton Applied Research, la conductividad encontrada a partir

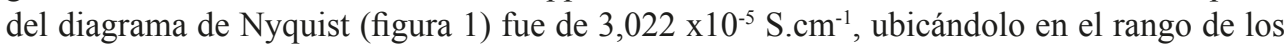
semiconductores ${ }^{5,6,7}$. La celda electroquímica consistió, en un sistema donde el tricapa queda en medio de dos placas de acero inoxidable, un lado de la placa se conectó al EA previamente cortocircuitado con el ER y el otro lado se conectó al EW. 


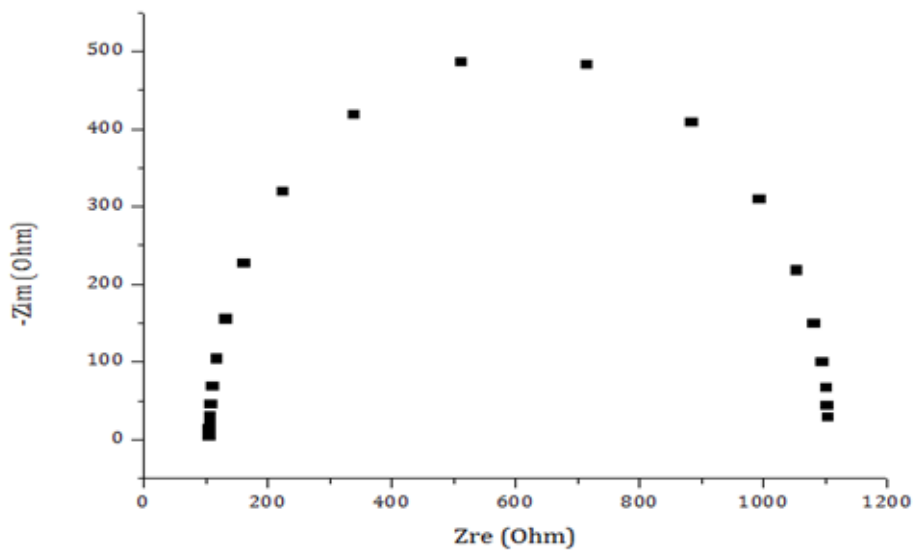

Figura 1. Diagrama de Nyquist del tricapa PPy/Biopolímero Conductor/PPy. El potencial de referencia fue el OCP (Open Circuit Potential) con un valor de -0,022 V.

Por otro lado, se realizó un análisis con la técnica de voltametría cíclica (VC) del dispositivo tricapa con área de $4,5 \mathrm{~cm}^{2}$. Este estudio se llevó a cabo mediante 4 rangos de potencial, de $-1 \mathrm{~V}$ a $1 \mathrm{~V},-2 \mathrm{~V}$ a $2 \mathrm{~V},-3 \mathrm{~V}$ a $3 \mathrm{~V}$ y $-4 \mathrm{~V}$ a $4 \mathrm{~V}$. En los pulsos de $-1 \mathrm{~V}$ a $1 \mathrm{~V}$ y $-2 \mathrm{~V}$ a $2 \mathrm{~V}$ no se observó proceso redox. En tanto que para los últimos rangos de potencial (-3 V a $3 \mathrm{~V}$ y -4 $\mathrm{V}$ a $4 \mathrm{~V}$ ), en la onda de oxidación se observaron bifurcaciones a $2 \mathrm{~V}$ y $3 \mathrm{~V}$, con corrientes de 2 y 3 mA (figura 2), además de dos picos bien definidos (pico 1 y pico 2), correspondientes a dos procesos de reducción del $P P y^{1}$, las bifurcaciones pueden deberse a un fuerte proceso de oxidación de las películas de $P P y$ asociada con ruido electrónico. La celda electroquímica empleada en este sistema es similar a la usada en el análisis de EIS.

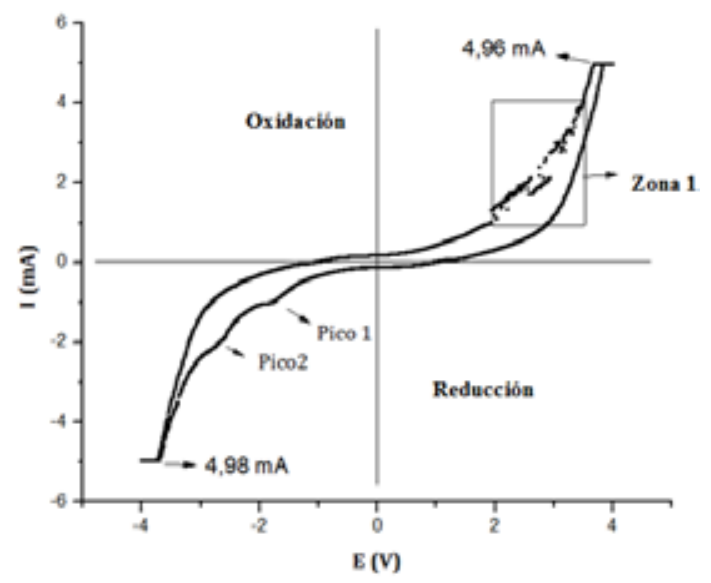

Figura 2. Voltagrama del tricapa en el rango de potencial de $-4 \mathrm{~V}$ a $4 \mathrm{~V}$, medida a $0,1 \mathrm{~V} \mathrm{~s}^{-1}$. El ER se cortocircuitó con el EA y se conectó a un lado de la placa de acero inoxidable y EW al otro terminal, el tricapa permaneció en medio de las dos placas. 


\section{Estudio motriz del tricapa}

Por otra parte, las técnicas de VC e impedancia, permitieron conocer que el dispositivo desarrollado es conductor de la electricidad y en él ocurren procesos de óxido/reducción característicos en los músculos naturales. Estos procesos de oxidación y reducción en las capas externas de $P P y$ se traducen en un cambio conformacional de su volumen generando movimiento. Los cambios de volumen en el tricapa son debidos al desplazamiento iónico producido durante la reacción electroquímica.

En la figura 3 se presenta un esquema de movimiento del tricapa que responde a estímulos eléctricos en el aire, en donde se presentan las distribuciones de los iones perclorato durante el proceso redox.

Al aplicar un pulso galvánico recurrente en los extremos del tricapa, el dispositivo se desplaza en sentido antihorario (figura 3a) y horario (figura 3b). Esto es: en el sentido horario la cara 1 de $P P y$ se reduce (disminuye su volumen), mientras que la cara 2 se oxida (aumenta su volumen), por lo contrario para el sentido antihorario se tiene una reducción en la cara 2 y una oxidación en la cara 1. Cabe resaltar que la oxidación corresponde al ingreso de los iones perclorato, provenientes de la matriz polimérica del biopolímero de almidón de yuca (electrolito sólido) hacia la cara de $P P y$. El mecanismo de intercambio iónico no ha sido actualmente definido con claridad, sin embargo, en la literatura8,9 han sido propuestos algunos mecanismos de intercambio, uno de los más aceptados explica las bases de la respuesta electroquímica, según lo representado por las ecuaciones 1 y 2.

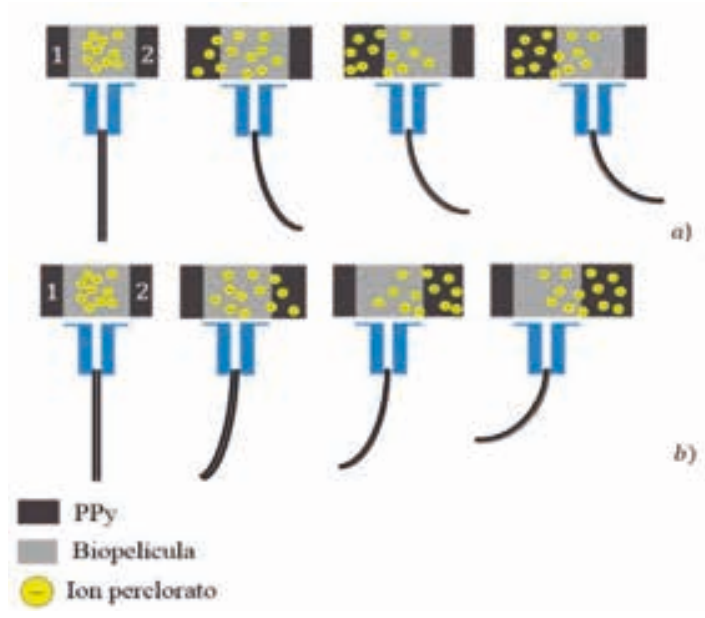

Figura 3. Esquema del movimiento del tricapa PPy/Biopolimero/PPy en el aire, las caras del dispositivo han sido numeradas como 1 y 2 . 
En el primer proceso de óxido/reducción, por razones de electronegatividad, la película de $P P y$ se comporta como un intercambiador de cationes $\left(\mathrm{C}^{+}\right)$:

$$
\left[P P y / \mathrm{X}^{-} / \mathrm{C}^{+}\right] \stackrel{e^{-}}{\leftrightarrow}\left[P P y^{+} / \mathrm{X}^{-}\right]+\left[\mathrm{C}^{+}\right]
$$

La reacción redox que conduce al segundo proceso de óxido/reducción puede ser descrito como sigue:

$$
\left[P P y^{++} / \mathrm{X}^{-}\right]+\left[\mathrm{A}^{-}\right] \stackrel{e^{-}}{\leftrightarrow}\left[\mathrm{PP}^{++} / \mathrm{X}^{-} / \mathrm{A}^{-}\right]
$$

Donde $\mathrm{X}^{-}$y $\mathrm{A}^{-}$representan los contraiones y los aniones, respectivamente. En este caso la electroactividad del PPy hace que la película se comporte como un intercambiador de iones. Esto quiere decir que los aniones de perclorato, provenientes del biopolímero de almidón de yuca que corresponde a un electrolito sólido, le proporcionan al $P P y$ los iones necesarios para mantener su electroneutralidad. La estructura del polímero adyacente $(P P y)$ es oxidada (pierde $e^{-}$) se abre, facilitando el paso a los iones, el proceso se invierte de manera continua dando como resultado un comportamiento electroquimiomecánico, típico de los músculos naturales.

En cuanto al análisis realizado, respecto al movimiento electroquimiomecánico del tricapa, se estudiaron los desplazamientos angulares en función del tiempo como respuesta a los pulsos eléctricos, obteniéndose así velocidades angulares de $\omega=0,039 \mathrm{rad} / \mathrm{s}$ en el sentido horario y $\omega=0,025 \mathrm{rad} / \mathrm{s}$ en el sentido antihorario, barriéndose ángulos de $52,2^{\circ}$ y $31,3^{\circ}$, respectivamente, lo cual corresponde a una amplitud de $83,5^{\circ}$.

\section{CONCLUSIONES}

Las proporciones empleadas de aglutinantes y el método experimental para la obtención de la biopelícula conductora, otorgaron propiedades de adherencia favorables para la construcción del tricapa, en donde la biopelícula se usó para desprender el $P P y$ del EW, además, la conductividad calculada fue de $3,022 \times 10^{-5} \mathrm{~S} \mathrm{~cm}^{-1}$ que la ubica en el rango de los semiconductores.

Con la técnica VC, se evidenció que el actuador tricapa, obedece a los procesos de oxidación/ reducción, en donde las capas de $P P y$ al ser oxidadas y/o reducidas producen un cambio significativo de su volumen que se traduce en un movimiento fluido del actuador en el aire en ambas direcciones.

Se encontraron algunas similitudes entre el músculo artificial desarrollado y los músculos naturales, entre las cuales se resalta el control sobre las velocidades y dirección de movimiento, la capacidad de mantenerse estable en un punto al cesar la corriente además de operar a bajos voltajes $(<4 \mathrm{~V})$. 
El dispositivo desarrollado se constituye como la quinta generación de músculos artificiales, debido a que funciona en aire, empleando un biopolímero conductor a base de almidón de yuca que actúa como puente intercambiador de iones entre las capas externas de PPy. Este dispositivo es el primero, reportado en el mundo que utiliza el almidón de cassava y es capaz de responder a estímulos eléctricos en el aire.

\section{AGRADECIMIENTO}

A la Fundación universitaria Los Libertadores, por su apoyo en la realización de esta investigación.

\section{REFERENCIAS BIBLIOGRÁFICAS}

1. Cortés Montañez MT. Músculos artificiales de tricapa, caracterización y diseño de dispositivos. [Tesis doctoral]. Universidad del país Vasco: Ciencias Químicas; 2002.

2. Otero TF. Polímeros conductores: Síntesis, propiedades y aplicaciones electroquímicas. Rev Iber Pol. 2003; 4: 1-37.

3. Chien Chu C, Wen Pin S, Pei Zen C et al. Onion artificial muscles. Appl Phys Lett. 2015; 106: 1-5. [Citado el 15 de sep 2015]. Disponible en: http://dx.doi.org/10.1063/1.4917498.

4. Parra DF, Tadini CC, Ponce P, Lugão AB. Mechanical properties and water vapor transmission in some blends of cassava starch edible films. Carbohyd Polym. 2004; 58: 475-481.

5. Arrieta Almario AA. Diseño de una red de sensores electro químicos inespecíficos basados en polímeros conductores para el análisis sensorial de vinos. [Tesis doctoral]. Universidad de Valladolid; 2004.

6. Díaz M, Rojas Giraldo A, Vera E, Cortés Montañez MT. Síntesis electroquímica y caracterización eléctrica de polímeros conductores a partir de pirrol. Rev Col Fis. 2005; 37: 357-360.

7. Arrieta Almario AA, Jaramillo A. Bioplásticos eléctricamente conductores de almidón de yuca. Rev Col Fis. 2014; 5: 42-49.

8. Arrieta Almario AA, Tarazona Cáceres RL. Study of kinetic formative and the electrochemical behavior of polypyrrole films. J Chil Chem Soc. 2009; 54: 14-19. Disponible en: http://dx.doi.org/10.4067/S0717-97072009000100004.

9. Otero TF. Los polímeros conductores como materiales biomiméticos. Bol Soc Esp Cerám y Vidrio. 1997; 36: 230-237. 